\title{
Mixing is required for uniform reconstitution of filter-dried protein antigens in a single-injection vaccine formulation
}

Article

Accepted Version

Creative Commons: Attribution-Noncommercial-No Derivative Works 4.0

Thangsupanimitchai, N. and Edwards, A. D. (2018) Mixing is required for uniform reconstitution of filter-dried protein antigens in a single-injection vaccine formulation. Vaccine, 36 (3). pp. 5058-5064. ISSN 0264-410X doi:

https://doi.org/10.1016/j.vaccine.2018.07.001 Available at https://centaur.reading.ac.uk/78129/

It is advisable to refer to the publisher's version if you intend to cite from the work. See Guidance on citing.

To link to this article DOI: http://dx.doi.org/10.1016/j.vaccine.2018.07.001

Publisher: Elsevier

All outputs in CentAUR are protected by Intellectual Property Rights law, including copyright law. Copyright and IPR is retained by the creators or other copyright holders. Terms and conditions for use of this material are defined in the End User Agreement. 


\section{CentAUR}

Central Archive at the University of Reading

Reading's research outputs online 


\title{
Mixing is required for uniform reconstitution of filter-dried protein antigens in a single-injection vaccine formulation
}

\author{
Napawan Thangsupanimitchai ${ }^{1}$, Alexander D. Edwards ${ }^{\star}$
}

Reading School of Pharmacy, University of Reading,

Whiteknights, Reading, RG6 6AP

*correspondence to a.d.edwards@reading.ac.uk

$<44>1183784253$

${ }^{1}$ Current address: Government Pharmaceutical Organisation, Bangkok, Thailand.

\begin{abstract}
Ambient temperature filter dried vaccine formulations have been proposed to simultaneously achieve thermostability and offer a ready-to-use immunisation device that combines reconstitution and injection. Vaccine concentration should be uniform at the point of injection, but the uniformity following direct reconstitution of filter-dried vaccines has not been reported. We present here a study of vaccine mixing and release following dissolution of filter-dried model protein and toxoid antigens within a single syringe, filter and needle unit. Release was better for filters made from glass than cellulose. Without additional mixing, uniformity was poor and only $41 \%$ of input protein was released from protein filter-dried onto glass fiber. In contrast, adding a simple glass bead and mixing by inversion, $100 \%$ release antigen solution was achieved, with uniform concentration at exit from the needle throughout a simulated injection. Adsorption onto alum adjuvant had no detectable effect on vaccine dissolution and mixing. The uniformity and yield of low doses of diphtheria and tetanus toxoid was also improved by mixing, albeit with a lower yield of $60-68 \%$. We conclude that uniformity and mixing should be studied to ensure safety and efficacy of directly reconstituted filter-dried vaccine formulations.
\end{abstract}

\section{Keywords}

Formulation; Filter dried; thermostabilised; mixing; adjuvant.

\section{Introduction}

A significant proportion of the cost of vaccination programs lies in safe refrigerated storage and distribution, and costs of trained healthcare professionals for administration. This has driven extensive research into drying and thermostabilisation technology for vaccines [1]. Traditionally freeze-dried vaccines still require refrigerated storage, although excipient optimization can achieve thermostable lyophilized vaccines [2]. Freeze-dried vaccines still require careful reconstitution and preparation prior to administration, typically reconstitution in a freeze-drying vial, followed by transfer to a syringe and needle for injection. Whilst the widespread use of liquid formulations distributed in pre-filled syringes for many common vaccines reduces staff time and skill level, liquid vaccine preparations are inevitably less stable than freeze-dried formulations, with solid formulations more stable and easier to handle [3, 4] because chemical (e.g. hydrolysis) and physical (e.g. foaming or aggregation) instability have far faster kinetics in aqueous form. Pre-filled syringes could be argued to be more expensive formulations than traditional lyophilized vials, but in reality freeze-drying is 
already a labour intensive and slow process constrained by capacity of industrial freeze-driers [3], and the processes used for manufacture of vaccines in pre-filled syringes are now well developed. Furthermore, lyophilized vaccine vials still require water for injection plus disposable syringe and needle, which whilst low-cost components, increase the complexity of the supply chain.

The ideal vaccine formulation must therefore noat only be produced from low cost components, but must also have cost-effective scalable manufacturing process, and the simplest possible format for the end-user administrating the vaccine dose. To give the product stability and avoid cold-chain storage, this device would contain a dry physical form produced using a scalable drying method which maximises thermostability. Whilst alternatives to injection such as dried oral solid dosage forms [5] offer benefits, the overwhelming majority of current vaccines require injection. Reconstitution of dried vaccine to a liquid for injection therefore becomes an important engineering consideration.

The cost of freeze-drying, and burden of cold-chain storage and complex preparation for administration has driven research into a range of novel formulations, and modelling has shown that thermostable vaccines would deliver significant improvements to the vaccine supply chain[6]. Multiple alternatives to freeze-drying have been explored, such as spray- or foam- drying [7]. A longestablished and intensively studied example is the use of ambient temperature drying to form an amorphous sugar glass from high glass transition temperature carbohydrates such as trehalose [8$10]$. This method has been combined with simplified formulation to deliver a thermostable, singlecomponent vaccine formulation and injection format that shows great promise $[11,12]$. The vaccine is dried to form a sugar glass on a non-woven fibre filter substrate, which is then encased in a filter housing which is connected between a syringe filled with water for injection, and the needle [13, 14]. The sugar glass vaccine dissolves in flow as the syringe is depressed, and the dissolved vaccine injected directly into the patient. This approach is suitable to fragile live attenuated recombinant viral vaccines including modified vaccinia and adenovirus, which were preserved on the membranes for at least 6 months storage at high temperature $\left(45^{\circ} \mathrm{C}\right)$ [13]. Similarly, hepatitis B vaccine dried onto filters are stable and immunogenic at $55^{\circ} \mathrm{C}$ for 7 weeks [9].

As this highly promising "all-in-one" formulation is now being developed for use in new human vaccine products, we questioned if the dissolution characteristics of reconstitution from the filter has been studied in enough detail. The uniformity of any drug prior to injection into patients is critical to safe and controlled administration and function, and we wished to understand if this novel formulation can deliver a uniform concentration of reconstituted vaccine. Whilst fluid flow through the filter matrix will generate a degree of turbidity, and some mixing will occur between reconstitution from the filter and the end of the needle, there is no mechanism driving axial redistribution if flow is unidirectional from syringe, through filter, into needle.

The filter units reported for thermostable filter-dried viral vaccines [13] are not freely available, and no design or specification has been published. Therefore, to study the efficiency of reconstitution from ambient temperature filter-dried vaccine formulations, we developed a simple model formulation using an unmodified plastic syringe, which made mixing and reconstitution easy to monitor and quantify. Likewise, complex and fragile live attenuated vaccines are expensive and challenging to visualize, making study of mixing and reconstitution unnecessarily complex. Tetanus and diphtheria toxins derived from Clostridium tetani and Corynebacterium diphtheriae, respectively are chemically inactivated to form toxoids that retain antigenic structure and potently induce a protective toxin-neutralizing immunity. Whilst very stable when compared to live attenuated whole viral vaccines, structure can still be lost on storage or during processing or formulation $[15,16]$ Toxoids such as tetanus toxoid are among the most heat-stable biological vaccine components. However, World Health Organisation (WHO) recommend that tetanus and diphtheria toxoids still require storage at $2-8^{\circ} \mathrm{C}$ for years, with reduced stability of months at $25^{\circ} \mathrm{C}$ and only weeks at $37^{\circ} \mathrm{C}$ [15]. In this initial proof-of-concept study, we opted to explore recovery and mixing using the simplest single-protein vaccine to permit simple visualisation, and therefore selected these two toxoids as a model to study reconstitution from filter-dried vaccines. 
We present here a pilot study of mixing following reconstitution of filter-dried protein antigens. We used an extremely simple and low-cost injection rig to explore the feasibility and effectiveness of active mixing within a syringe prior to injection. To permit not only precise quantitation of vaccine dissolution but also direct imaging of uniformity of vaccine solution, we studied fluorescently labelled protein and alum-adsorbed protein and diptheria and tetanus toxoid as model vaccine antigens. We found that the filter dried vaccine is unlikely to be mixed effectively following reconstitution by addition of water alone, and that additional mixing by addition of a glass bead combined with agitation not only increased uniformity of vaccine delivered, but increased the yield.

\section{Materials and Methods}

\subsection{Materials}

To make fluorescent model protein antigen, BSA at $>=2 \mathrm{mg} / \mathrm{ml}$ in $0.5 \mathrm{M}$ of carbonate buffer ( $\mathrm{pH} 9.5$ ) and $10 \mathrm{mg} / \mathrm{ml}$ FITC in anhydrous dimethyl sulfoxide (DMSO) were combined a ratio of 80-160 $\mu \mathrm{g}$ FITC per mg of antibody. After incubation at room temperature for 1 hour, conjugates were purified by size-exclusion chromatography. BSA, FITC and Sephadex G-50 superfine were from SigmaAldrich (Dorset UK). Phosphate buffer saline tablets (Sigma, Dorset UK) were dissolved in ultrapure water and ProClin 300 (Sigma, Dorset UK) was added as a preservative. Adjuvant adsorbed antigen was prepared following manufacturers direction, briefly Imject Alum (Thermo Fisher UK, Paisley UK) was added to FITC-BSA at a final volume ratio of alum to immunogen of 1:3, then mixed together for 30 minutes for efficient adsorption. Non-adsorbed diphtheria and non-adsorbed tetanus toxoid were used as model antigens in a simulated combined vaccine, and were obtained from the National Institute for Biological Standards and Control (NIBSC, Potters Bar, UK) as well as monoclonal and polyclonal antibodies for sandwich ELISA antigen quantitation; all were reconstituted following the supplier's recommended method. Two types of filter were compared for filter drying of model vaccine antigens: cellulose filters were Whatman ${ }^{\mathrm{TM}}$ qualitative filter papers grade 1 (Cat No 1001-055) with $180 \mu \mathrm{m}$ thickness; glassfibre filters were grade 8964 with $429.3 \mu \mathrm{m}$ thickness (Ahlstrom, Lyon, France). Glass beads for mixing were taken from $1.2 \mathrm{~mL}$ Sodium Heparin S-Monovette® blood collection tubes (Sarsedt, Leicester UK).

\subsection{Preparation of ambient temperature dried model antigen filters and combined injection device}

A simple prototype combined injection device was made using two filter-dried vaccine antigen squares loaded into the barrel of a $2 \mathrm{ml}$ plastic disposable syringe (BD, Berkshire UK). To promote mixing, a $3 \mathrm{~mm}$ diameter glass mixing bead taken from a Monovette ${ }^{\circledR} 1.2 \mathrm{~mL}$ blood collection tube (Sarstedt; Leicester UK) was added with the filters (Fig. 1A). To make filter-dried antigen squares, $10 \mu \mathrm{L}$ of fluorescein, fluorescent protein or antigen solution was added per $5 \mathrm{~mm}$ square filter, and dried at ambient temperature (typically $20^{\circ} \mathrm{C}$ ) in a petri dish until completely dry, which took a minimum of 1 hour (drying time was based on previous thermogravimetric studies of vaccine drying onto polymer films [17], and confirmed by kinetic analysis of mass loss). To establish methods and directly image reconstitution, $1 \mathrm{mM}$ sodium fluorescein solution was dried onto filters. This was followed by study of filters loaded with fluorescently labelled BSA, used as a model protein to more accurately reflect vaccine antigen dissolution. A $3.2 \mathrm{mg} / \mathrm{mL}$ solution of BSA-FITC was found to be the lowest concentration suitable for quantitative imaging in the chamber. To determine if a real toxoid vaccine antigen behaved similarly to the high concentration of model protein used, both diphtheria and tetanus toxoids were diluted in ultrapure water to the lowest concentration that permitted quantitation by ELISA. A concentration of $4 \mathrm{lf} / \mathrm{ml}$ was selected for both toxoids mixed in a ratio of $1: 1$, and $10 \mu \mathrm{L}$ was pipetted on a $5 \times 5 \mathrm{~mm}$ square of filter to yield 0.02 If of diphtheria and tetanus toxoid per filter. Toxoid concentrations are presented in flocculation value per $\mathrm{ml}(\mathrm{If} / \mathrm{mL})$. When two filters in a syringe were reconstituted with $500 \mu \mathrm{l}$ ultrapure water, the maximum concentration of reconstituted vaccine expected was $0.08 \mathrm{lf} / \mathrm{mL}$. 


\subsection{Measurement of mixing and dissolution of filter dried model vaccine antigens}

To observe fluorescent protein mixing directly after reconstitution, filters with dried $1 \mathrm{mM}$ sodium fluorescein were imaged in a dark box using blue light excitation through an amber acrylic emission filter (IOrodeo, San Diego, USA) using a Canon S120 digital camera using manual aperture and exposure control. To observe fluorescent protein concentration delivered through the needle during a simulated vaccine injection, a chamber was made between a black acrylic and a clear polycarbonate sheet using two layers of pressure-sensitive adhesive tape to form a $0.2 \mathrm{~mm}$ gap (Fig $2 \mathrm{~A})$. The tape was cut to form a shape that allowed the solution to be imaged within a $20 \mu \mathrm{L} 2 \mathrm{~mm}$ high and $10 \mathrm{~mm}$ long rectangular space immediately after leaving the needle. Following addition of $0.5 \mathrm{~mL}$ water with or without mixing, the syringe was attached to a $25 \mathrm{~mm}$ long 23 Gauge needle, bent through 90 degrees and connect to the imaging chamber. As the syringe plunger was depressed the chamber was imaged using the camera, dark box, and excitation source plus emission filter described above. The syringe barrel was imaged to define volume delivered. A dried filter with standard dose of fluorescein was included beside the chamber to permit comparison of intensity between different tests; in all tests the intensity of this standard sample was equal, confirming excitation intensity and camera sensitivity was reproducible. All videos and images were processed to evaluate uniformity and concentration of released protein using ImageJ [18].

After the syringe was completely emptied the total yield of model antigen in the $\sim 0.5 \mathrm{~mL}$ solution delivered was measured using three methods. Firstly, release of BSA-FITC with or without adsorption onto alum was measured using protein determination with the Pierce $660 \mathrm{~nm}$ Protein Assay following manufactures instructions. Briefly, $10 \mu \mathrm{L}$ samples were mixed with $150 \mu \mathrm{l}$ of Pierce 660 Protein Assay Reagent (Thermo Fisher Scientific) in a microplate, and after incubation at a room temperature for 5 minutes the absorbance at $660 \mathrm{~nm}$ recorded and compared to a standard curve prepared from known BSA or FITC-BSA samples. When adsorbed onto alum adjuvant, protein concentrations were calculated from a standard curve of alum-adsorbed BSA. Secondly, absorbance at 495 was measured in parallel to determine fluorescein concentration; the release profiles were very similar to that observed for protein content. Thirdly, total antigen release from filter-dried toxoid squares was measured by sandwich ELISA using reported protocols for diphtheria [19] and tetanus [20] with modified blocking (2\% BSA) and substrate (SigmaFast OPD; Sigma, Dorset UK). The antidiphtheria monoclonal capture antibody was NIBSC code: 10/130, and guinea pig polyclonal detection antibody was NIBSC code: 10/128. For tetanus, monoclonal capture antibody was NIBSC code: 10/134 and guinea pigs polyclonal detection antibody was NIBSC code: 10/132. Where indicated, a two-sample students t-test was used to assess statistical significance, and statistically significant differences where $p<0.05$ marked with *.

\section{Results and discussion}

\subsection{A uniform flow of protein requires mixing}

We initially used fluorescein dye, BSA-FITC conjugates and toxoid antigens to study the mixing and uniformity following direct reconstitution from filters in a simple reconstitution device. When imaged immediately after addition of ultrapure water to filter-dried fluorescein we found clear variations in intensity, in contrast to a bright and homogenous solution after vigorous mixing in the syringe (Fig. 1). This indicated that an additional mixing step should be incorporated to ensure vaccine is homogeneous on injection. Since mixing can occur in the syringe and needle during injection, and mixing is hard to quantify within the syringe body, an imaging chamber was made to measure fluorescence directly as the solution exits a needle suitable for vaccine administration (Fig. 2A). Concentration was quantified within a uniform rectangular $20 \mu \mathrm{L}$ chamber that was $2 \mathrm{~mm}$ high, $10 \mathrm{~mm}$ long, with a thickness of $0.2 \mathrm{~mm}$. Glass beads are used to mix anticoagulant into small volume blood samples in products such as Monovette ${ }^{\circledR}$. In pilot tests, the effectiveness of adding a glass mixing bead, taken from a $1.2 \mathrm{~mL}$ Monovette $\AA$, and mixing by inversion was assessed with filterdried fluorescein dye. Inversion mixing with a glass bead gave a higher average fluorescence intensity and smaller standard deviation than inversion without the bead (Fig 2B), and the bead was 
therefore included in all subsequent experiments. Pilots also confirmed that dissolution from filters differs somewhat between the small molecule dye fluorescein vs protein, and so subsequent experiments used fluorescently labelled model protein.

BSA-FITC was chosen as a model protein antigen and filter-dried onto cellulose and glass fibre squares. The release profile was assessed by measuring intensity of sequential $50 \mu \mathrm{L}$ volumes during injection through the chamber, after reconstituting with or without mixing. Both uniformity of intensity and overall yield were improved by mixing. Without mixing, there was significant variation both between replicates, and in the concentration delivered per unit volume during the injection period (Fig. 2C). Spikes in concentration were often detected for individual samples (for example in the first and/or last volume). This variation was reduced following mixing for any length of time, for both cellulose and glass filters (Fig. 2C-F). With glass filters, mixing also gave a clear increase in intensity. Although mixing also improved uniformity for cellulose filters, the fluorescence intensity was somewhat lower than glass filters, suggesting less efficient release. When mean and standard deviations were plotted, the reduction in variance and increase in released concentration were clear throughout the duration of the simulated injection (Fig. 2G). Whilst this study of a simple model protein antigen is highly informative, further study would be required to determine mixing efficacy for larger and more complex vaccine formulations including live attenuated vaccines which have been shown previously to be fully dry and achieve long-term thermostablisation after filter drying [13].

\subsection{Maximal yields are obtained from glass filters mixed by inversion with glass bead}

Whilst the imaging system was ideal to determine uniformity in concentration as the injected volume passes out of the needle, and showed that variation was eliminated by mixing, it was important to determine independently the overall protein yield and determine if the apparent increase in concentration following mixing could be confirmed. The entire $500 \mu \mathrm{l}$ injection was therefore withdrawn from the reservoir, and protein yield measured. This clearly showed that the imaging system underestimated the increase in protein release following mixing, and also confirmed that the best yield was achieved by a combination of glass filters and mixing (Fig 3A). No further benefit was seen increasing mixing time above 1 minute. These observations were confirmed by absorbance measurements to quantify fluorescein concentration in bulk solutions recovered after complete injection. Likewise, when calibrated against standard concentrations imaged in the same chamber, similar total concentrations were estimated from the fluorescent imaging chamber to that found by bulk protein determination (data not shown). This validated the findings from the fluorescent imaging system, demonstrating that direct visulisation of mixing following reconstitution can be a valuable tool to optimize the uniformity of vaccine delivered from novel formulations and/or injection devices.

Protein antigens are typically weakly immunogenic without adjuvant; it was therefore important to assess the effect of the adjuvant on filter-dried protein release. After the fluorescent protein was adsorbed to aluminium hydroxide adjuvant using standard methodology, the same protein measurement procedure was performed and once again significantly lower protein concentration was recovered without mixing. The analytical reference in this experiment was known concentrations of protein adsorbed to alum, to ensure that the aluminium hydroxide was not interfering with protein determination assay. Again, a high yield of alum-adsorbed fluorescent protein was released with mixing by inversion with a glass bead (Fig 3B). Although a slightly low protein concentration was observed in the three-minute mixing group than a control filter that had not been dried permitting maximal reconstitution, no significant difference was found between this and control group. Whilst this suggested that alum adjuvant did not influence reconstitution of this specific model fluorescent protein, different vaccine antigens, adjuvants, and formulations will require individual optimization of mixing on reconstitution, using the systems developed here.

\subsection{Mixing is required for maximal recovery of low concentration filter-dried toxoid antigen}

Antigen concentration for some subunit vaccines is substantially lower than that used in the imaging studies, making it far more challenging to either visualize protein release or measure protein content 
directly. Toxoid vaccines are potent immunogens and so very low concentrations are formulated in diphtheria and tetanus vaccines (typically around 1 If per dose), and the reference toxoid samples we used in this study were supplied with a high concentration of protein carrier, further complicating measurement. The low concentrations plus the presence of protein carrier made it challenging to label for direct imaging or to measure antigen concentration and uniformity by fluorescence or protein determination. Instead, we exploited sandwich immunoassays developed specifically for quantitation of antigenically intact toxoids for quality control. The high analytical sensitivity of sandwich ELISA permitted us to test toxoid concentrations at or below that required for human vaccine dose. A very low-dose diphtheria and tetanus combined toxoid vaccine prototype incorporating glass fiber filterdried antigen samples was prepared and tested and antigen content evaluated by sandwich ELISA. Significant variations in antigen content were found in the unmixed group for both diphtheria and tetanus toxoids (Fig. 4). Although an increased yield with lower sample-sample variation was observed with mixing, the yield was still substantially lower than that observed with BSA-FITC (Figs. 3 and 4). Approximately $60-70 \%$ of total input antigen content was recovered after five minutes of mixing, compared to the control sample which was treated identically but without filter drying and diluted in ultrapure water to produce a $500 \mu \mathrm{L}$ sample. One explanation was that at this very low concentration of antigen, significant protein was irreversibly adsorbed onto the glass fiber filter during drying, which could potentially be avoided by pre-blocking or addition of blocking excipients. Specific formulations must be designed for each and every antigen or vaccine developed, and the addition of excipients such as surfactants may reduce loss by adsorption onto glass bead, filter, or syringe. Likewise, adsorption of the toxoid onto alum adjuvant might overcome this challenge. Another possibility is that the drying and reconstitution process denatured part of the toxoid antigen, rendering it undetectable in the sandwich ELISA that is designed to recognize antigenically intact toxoid. However, the improved uniformity and increased yield with mixing confirmed our observations with high dose fluorescent model protein that mixing is important for uniformity and yield.

\subsection{Suitability of simple combined filter plus bead injection concept}

This demonstration of a need for mixing for both maximizing recovery and ensuring injected vaccine is uniform after reconstitution illustrated the value of our simple combination of filter-dried vaccine plus glass mixing bead within a syringe as a model system. Figure 1B shows clearly that both yield and uniformity are improved by addition of a glass bead when filters are mixed. However, significant optimization and modification would be required for this to be used as a complete vaccine delivery system. Firstly, the mixing bead prevents complete depression of the syringe plunger, leaving a significand dead volume. This could be avoided by use of custom syringe design. Secondly, design for manufacture is required to ensure cost-effective production and packaging, taking into account the need for sterile manufacture and aseptic processing. Thirdly, whilst glass mixing beads are safe for use within plastic Monovette $\AA$ syringe, if glass syringe was required e.g. for moisture protection, a plastic bead would likely be required to avoid the risk of glass bead scratching the glass syringe internally. Finally, a major design aspect is protecting the dried dose from moisture whilst incorporating water for injection within a package for distribution. One option is for waterimpermeable packaging (e.g. foil sealed pouch) for the dried vaccine, plus completely separate water for injection. Alternatively, if a single device combines dried vaccine with water for injection, waterimpermeable materials will be required to prevent water vapour penetrating the chamber where the filter dried vaccine dose is stored. There are no reports of uniformity of injected dose and mixing achieved when using the separate filter chamber reported by Alcock et al [13], and the lack of design details for these devices prevents us speculating if a mixing bead could readily be incorporated within such a device. These engineering challenges must be solved for any such 'all-in-one' vaccine delivery system to be safe for human use. However, this simplified model system was not intended as a fully developed vaccine formulation, but proved an informative testbed to demonstrate the importance of the neglected area of mixing and uniformity following reconstitution from dried vaccines. 


\section{Conclusions}

In conclusion, we suggest that direct reconstitution and injection of protein antigens from a sugar glass dried at ambient temperature onto a filter does not deliver a uniform solution, and may not be appropriate for vaccine injections. We observed fluctuations and inconsistency in fluorescence intensity in the unmixed solution both within the syringe barrel (Fig 1A) and on exiting the needle during simulated injection (Fig 2). In contrast, after even 1 minute of mixing by inversion with a glass mixing bead a decreased standard deviation of solution exiting the needle was achieved, combined with an increase in protein antigen yield (Fig 2-4). This observation suggests that efficiency of mixing and uniformity of delivered dose should be evaluated for 'all-in-one' vaccine delivery devices that directly reconstitute filter-dried vaccine during injection; whilst the thermostability of these formulations has been thoroughly investigated [13] our findings here strongly suggest it is vital to ensure that this thermostability is matched with uniform and reliable reconstitution to avoid possible variation in efficacy in vivo. Surprisingly, adsorption onto alum adjuvant had no measurable effect on reconstitution and recovery of our model antigen (fluorescently labelled BSA). Recovery of toxoids dried at very low concentrations (representative of some potent human vaccines) was however lower than yields of fluorescently labelled BSA dried in microgram quantities, even with mixing. This suggested that each vaccine requires individual optimization to ensure effective recovery. We found a $2 \mathrm{ml}$ disposable syringe can be used as a simple and low-cost housing for filter-dried vaccine, if a $3 \mathrm{~mm}$ glass bead is included and the device is mixed by inversion following addition of water for reconstitution. This simple single unit device would permit simpler reconstitution and distribution than conventional vial lyophilized doses. However, significant further engineering and design-formanufacture is now required, combined with full evaluation of vaccine delivery efficacy in vivo, before this simple unit could be utilized. This pilot investigation suggests that reconstitution dynamics should be scrutinized for all single-injection devices intended to exploit direct reconstitution of thermostable dried vaccines.

\section{FIGURE LEGENDS}

Figure 1: Lack of uniformity of reconstituted fluorescein solution without mixing. $10 \mu \mathrm{L} 1 \mathrm{mM}$ sodium fluorescein was dried onto filters, and placed in the barrel of a $2 \mathrm{ml}$ syringe $(A)$ and imaged as $0.5 \mathrm{ml}$ of ultrapure water was added $(B)$. The syringe was then mixed by vigorous shaking, showing uniform fluorescence when well mixed (C).

Figure 2: Mixing by inversion with glass bead gives uniform delivery at needle exit of model protein antigen from filter-dried model vaccine dose. A Diagram and example image of injection apparatus to measure protein concentration at needle exit during injection. B Effect of glass bead on mixing for sodium fluorescein. C-F Three individual replicate plots of BSA-FITC intensity at needle exit during $500 \mu \mathrm{L}$ injection from cellulose and glass filters with no mixing $(\mathrm{C})$ or the indicated duration of inversion (D-F). G Comparison of mean and standard deviation of triplicates showing increased intensity and reduced variance with mixing vs no mixing. Error bars indicate $+/-$ standard deviation of three replicate tests.

Figure 3: Mixing increases yield from filter-dried model protein antigen. A The entire $500 \mu \mathrm{L}$ volume imaged in Fig 2 was collected for BSA-FITC samples, and antigen yield measured by protein determination. B BSA-FITC was adsorbed onto alum antigen and dried onto glass filters, followed by assembly into prototype single-injection devices and reconstitution with or without mixing as indicated. Total antigen yield was measured in bulk $500 \mu \mathrm{L}$ volume by protein determination. The line indicates maximal possible recovery and is the protein concentration from a control non-dried sample of BSA-FITC diluted directly into $500 \mu \mathrm{L}$ alongside filter reconstitution. Bars indicate mean and error bars 1 standard deviation of three independent replicates. * indicates statistically significant differences $(p<0.05)$ calculated using a student T-test.

Figure 4: Mixing increases yield and reduces variability of release of low dose toxoid antigen, but with lower recovery. $10 \mu \mathrm{L}$ of a $2 \mathrm{lf} / \mathrm{mL}$ diptheria and tetanus toxoid mixture was dried per filter. 
This dose was the lowest concentration that permits quantitative measurement of release by sandwich ELISA, at this concentration a human vaccine dose would be $0.5 \mathrm{~mL}$. Each bar presents mean and standard deviation of triplicate ELISA measurements for one syringe containing 2 filters plus a mixing bead. The control sample is an identical filter plus $10 \mu \mathrm{L}$ toxoid solution, which was not dried but directly diluted to $500 \mu \mathrm{L}$ in parallel; the theoretical maximum concentration with complete release would be $0.08 \mathrm{lf} / \mathrm{mL}$.

\section{Acknowledgements}

NT was supported by the Government Pharmaceutical Organisation (GPO), Bangkok, Thailand.

\section{References}

1. Kristensen, D., D. Chen, and R. Cummings, Vaccine stabilization: research, commercialization, and potential impact. Vaccine, 2011. 29(41): p. 7122-7124.

2. Amorij, J.P., et al., Rational design of an influenza subunit vaccine powder with sugar glass technology: Preventing conformational changes of haemagglutinin during freezing and freezedrying. Vaccine, 2007. 25(35): p. 6447-6457.

3. Walters, R.H., et al., Next Generation Drying Technologies for Pharmaceutical Applications. Journal of Pharmaceutical Sciences, 2014. 103(9): p. 2673-2695.

4. Wang, W., Lyophilization and development of solid protein pharmaceuticals. International Journal of Pharmaceutics, 2000. 203(1-2): p. 1-60.

5. Mercier, G.T., et al., Oral immunization of rhesus macaques with adenoviral HIV vaccines using enteric-coated capsules. Vaccine, 2007. 25(52): p. 8687-8701.

6. Lee, B.Y., et al., The Impact of Making Vaccines Thermostable in Niger's Vaccine Supply Chain. Vaccine, 2012. 30(38): p. 5637-5643.

7. Ohtake, S., et al., Heat-stable measles vaccine produced by spray drying. Vaccine, 2010. 28(5): p. 1275-1284.

8. Crowe, L.M., D.S. Reid, and J.H. Crowe, Is trehalose special for preserving dry biomaterials? Biophysical journal, 1996. 71(4): p. 2087-2093.

9. Roser, B., Stable liquid vaccines and drugs for the 21st century. Future Microbiology, 2006. 1(1): p. 21-31.

10. Ricker, J.V., et al., Trehalose maintains phase separation in an air-dried binary lipid mixture. Biophysical journal, 2003. 84(5): p. 3045-3051.

11. De, C.S., B. Roser, and S. Sen, Pharmaceutical device for the administration of substances to patients, in Google Patents. 2007: WO2007057717.

12. Scherließ, R., Delivery of antigens used for vaccination: recent advances and challenges. Therapeutic delivery, 2011. 2(10): p. 1351-1368.

13. Alcock, R., et al., Long-Term Thermostabilization of Live Poxviral and Adenoviral Vaccine Vectors at Supraphysiological Temperatures in Carbohydrate Glass. Science Translational Medicine, 2010. 2(19).

14. Dhapte, V., et al., Alternatives for Refrigerated Vaccines: A Contemporary Prerequisite. International Journal of Pharmacy and Pharmaceutical Sciences, 2013. 5(3): p. 99-102.

15. Milstien, J., U. Kartoglu, and M. Zaffran, Temperature sensitivity of Vaccines. 2006, World Health Organisation: Geneva, Switzerland. p. 11-15.

16. McHugh, K.J., et al., Single-injection vaccines: Progress, challenges, and opportunities. Journal of controlled release : official journal of the Controlled Release Society, 2015. 219: p. 596-609.

17. de Barros, J.M.S., et al., A Laminated Polymer Film Formulation for Enteric Delivery of Live Vaccine and Probiotic Bacteria. Journal of Pharmaceutical Sciences, 2014. 103(7): p. 2022-2032.

18. Hartig, S.M., Basic image analysis and manipulation in ImageJ. Current protocols in molecular biology, 2013: p. 14.15. 1-14.15. 12. 
19. Coombes, L., et al., Development and use of a novel in vitro assay for testing of diphtheria toxoid in combination vaccines. Journal of Immunological Methods, 2009. 350(1): p. 142-149.

20. Coombes, L., et al., In vitro antigen ELISA for quality control of tetanus vaccines. Biologicals : journal of the International Association of Biological Standardization, 2012. 40(6): p. 466-472.

\section{Graphical Abstract}

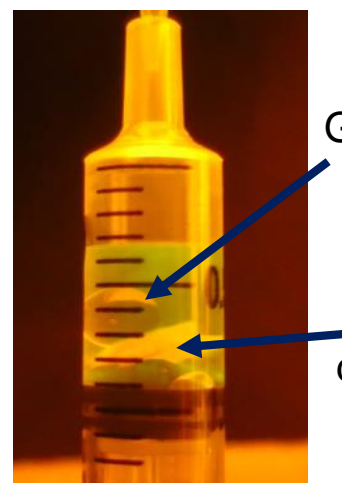

Glass bead

for mixing

$+$

Vaccine

dried onto

filter

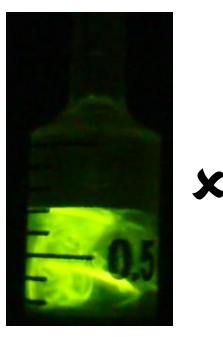

Reconstitute without mixingconcentration varies

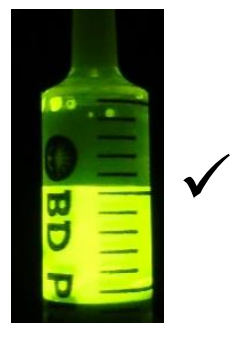

Mixing by inversion increases yield and delivers uniform solution

\section{Figures}
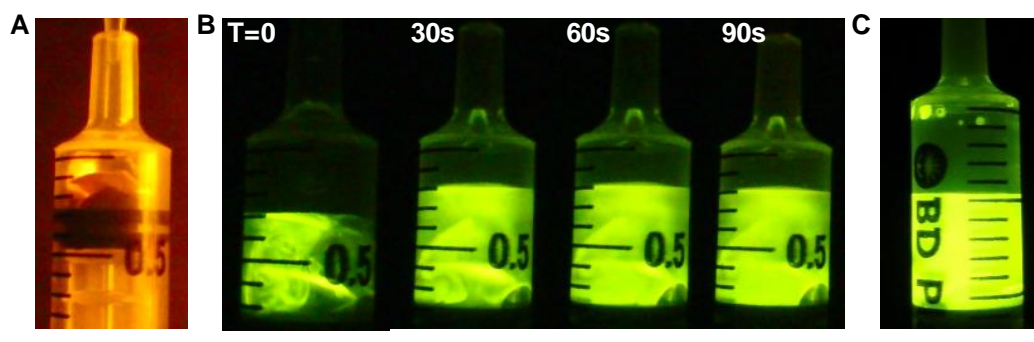

Figure 1 
A
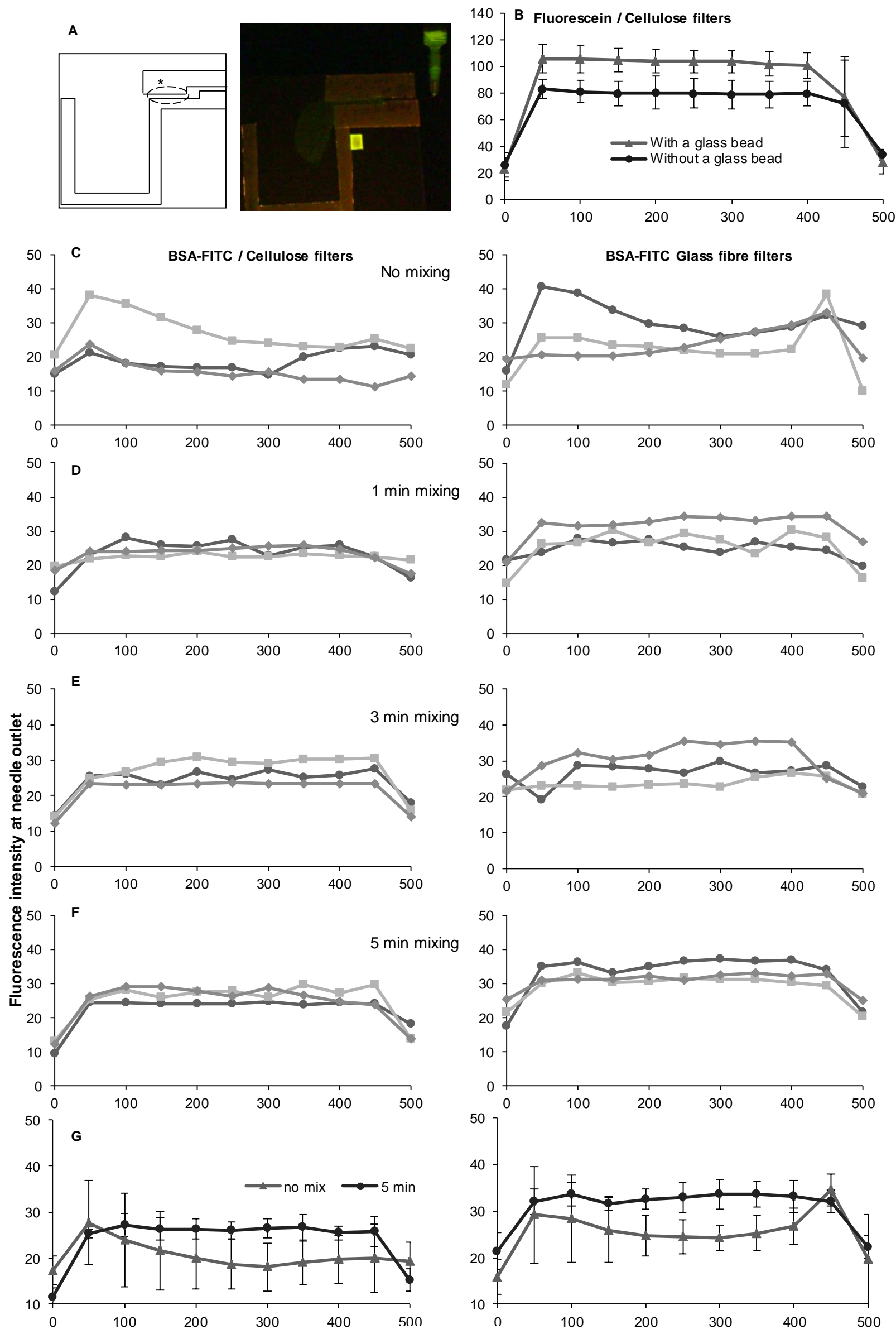
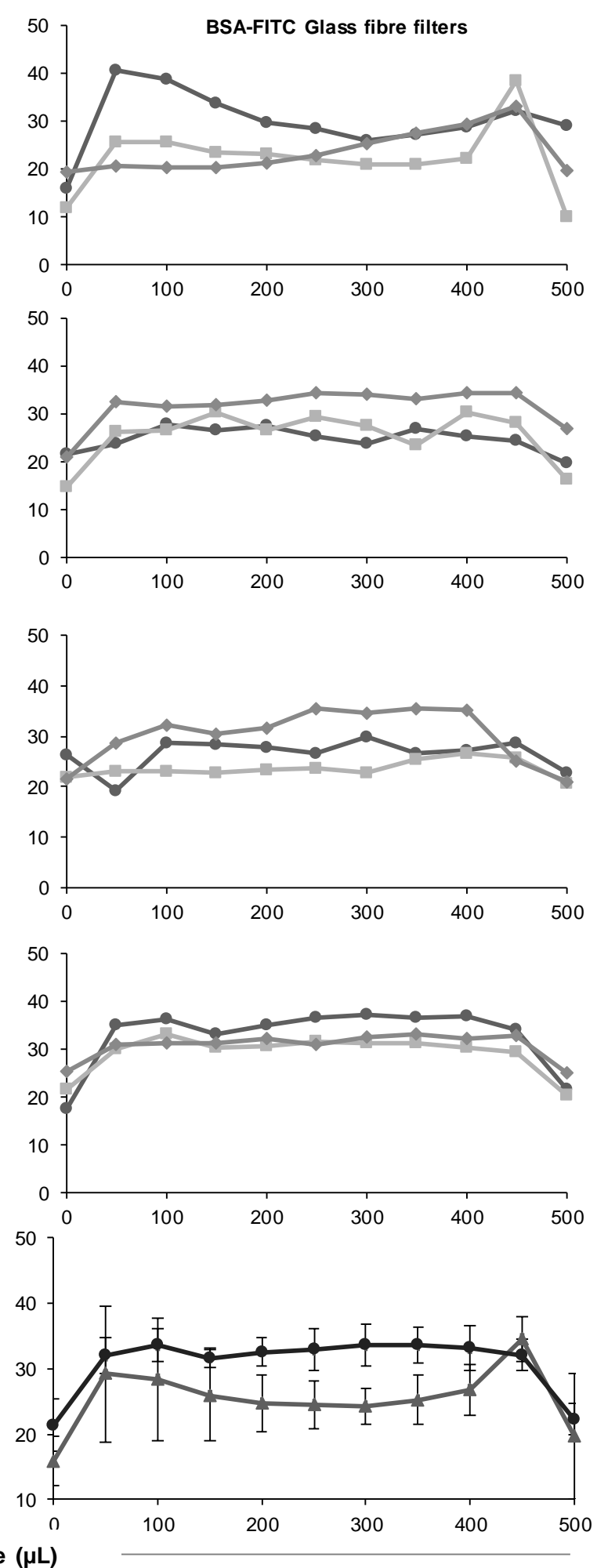

Figure 2 

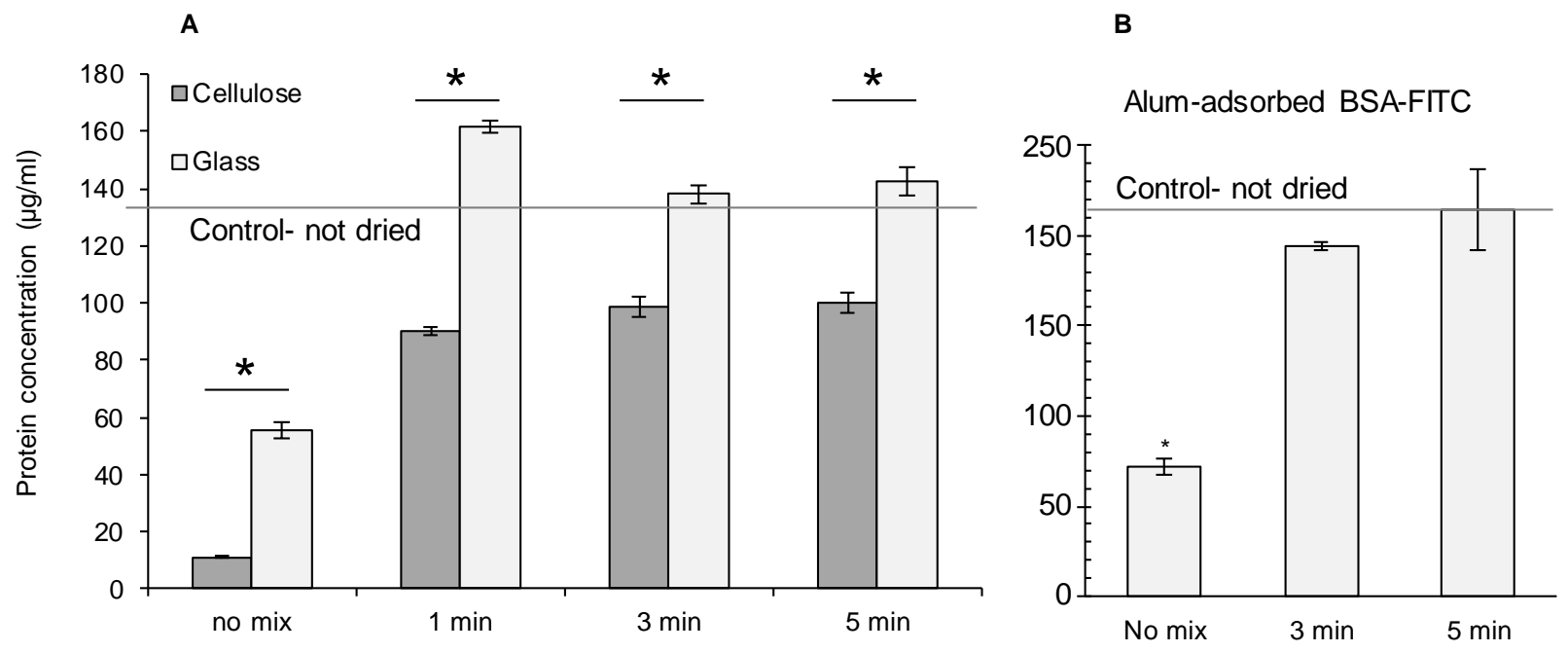

Figure 3

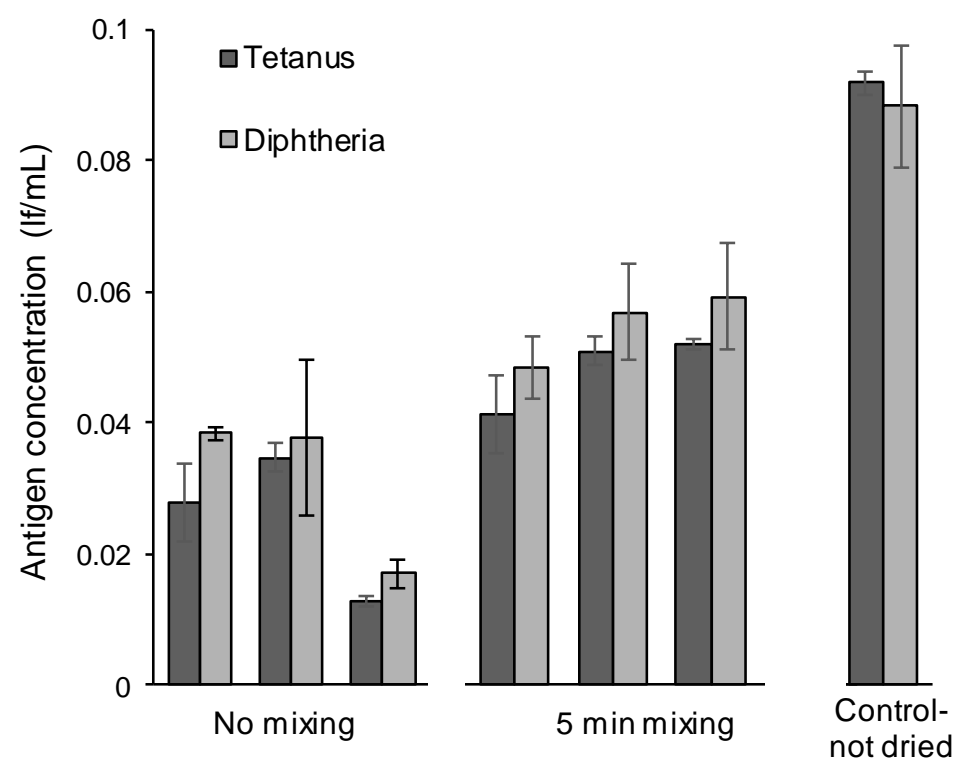

